\title{
Professionalism: Assessing physician behaviour
}

$\mathrm{S}$ ome things are easier to measure than others. Want to check your temperature? Grab a thermometer. Want to know your weight? Jump on a scale. Want to gauge how much your spouse actually likes your new toupee? That's a bit trickier.

This also holds true in the field of medicine. It's not difficult, for example, for a hospital to measure the number of patients it receives in a year or how much it spent on medical equipment. But what about the professionalism of physicians? Again, pretty tricky.

Of course, you can't hang a definitive score on a physician's conduct as if it were a clear-cut metric. Still, it can be assessed and valuable feedback can be provided to improve medical practice. It's not easy, but it's possible. It's also an important step in improving professionalism in medicine, which many doctors believe is regressing.

"Once you start to measure something, people start to value it more," says Dr. Tim Wilkinson, associate dean of medical education at the University of Otago in Christchurch, New Zealand.

There are a few important considerations a health care organization should make when assessing physician professionalism. A big one is context. Understanding the situation that gave rise to an incident of unprofessional behaviour may not justify it, but it will at least help explain why it occurred.

"Someone might be rude to a patient when there are 20 other patients waiting to be seen. It's not desirable but it's understandable. On the other hand, if that is the only patient seen all day, that's a different story," says Wilkinson. "The same behaviours have different meanings in different contexts."

Another salient factor is the time frame over which professionalism is assessed. It wouldn't be fair to pass judgment on a physician's professionalism based on behaviour observed over a short period. Rather, behaviour should be assessed over a long time in multiple settings under various conditions - during emergencies, during routine patient visits, when under stress, when on-call, while providing care alone, while collaborating with colleagues. Eventually,

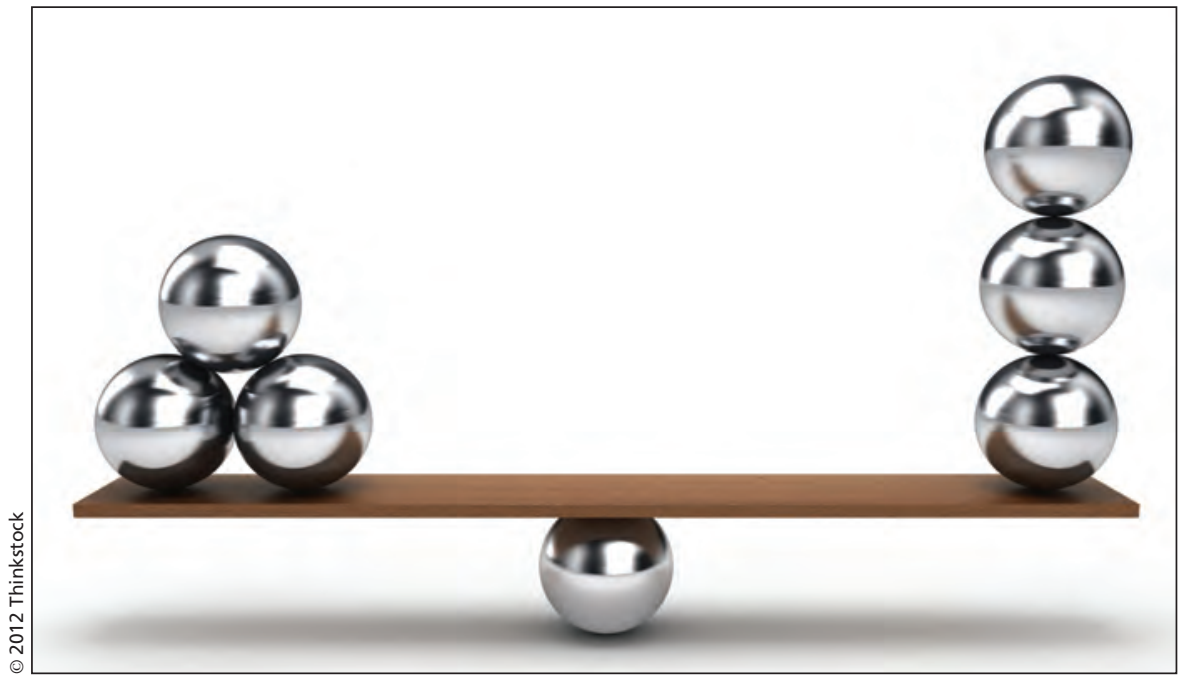

Experts say that a balance is needed in measuring professionalism, with allowances for mistakes and frailty.

an accurate picture of an individual's professionalism will emerge.

"The key point that people talk about with assessment of professionalism is that it has to be longitudinal," says Dr. Pier Bryden, a psychiatrist and the faculty lead in ethics and professionalism for undergraduate medical education at the University of Toronto in Ontario. "You have to look at it in a complex way over time."

It is also important that the assessment process be realistic. Doctors are only human, after all, and everyone makes mistakes. An egregious lapse in professionalism, such as sexually abusing a patient, would naturally have severe consequences. A curt response uttered during a bad day, on the other hand, is another story.

"One of the things that can happen is the creation of a very unrealistic definition of perfection that none of us can claim to meet," says Bryden. "You have to allow room for mistakes and frailty."

It would also be wise for health care organizations to incorporate the assessment process into daily activities. There are many unacceptable workplace behaviours that hardly require super sleuthing skills to uncover: showing up late for work, leaving early, failing to notify colleagues when absent for illness or other reasons. All too often, such behaviour is allowed to continue and goes undocumented.

"There are lots of opportunities in daily practice to note when someone is acting unprofessional," says Dr. Tim Allen, associate director of examinations for the College of Family Physicians of Canada. "The problem is that we don't note these behaviours."

Ferreting out unprofessional behaviour would be much easier by doing a better job of regularly documenting it, rather than relying on the memories of coworkers and patients at some date far removed from a particular incident. But it's important to approach such a process as a formative tool, says Allen, not a punitive one. "The whole point is not to crucify people, but to point things out to them so they can change."

Various processes and tools have been introduced over the years for the purpose of assessing professionalism. Still, much work remains to be done in this area, Wilkinson and colleagues have noted: "We see professionalism as central to the practice of medicine, yet the difficulty of its assessment is nearly great as the value we place on it" (Acad Med 2009;84:551-8).

Still, they forged ahead, discovering that they had to overcome two major problems in their quest to create a "blueprint to assess professionalism." The first was going through the many definitions of professionalism in medical literature, parsing out the core elements and breaking them down into measureable components (www.cmaj.ca/lookup/doi/10.1503 
/cmaj.109-4211). The second problem was that most previous attempts to assess professionalism had resulted in the development of single tools, which have proven inadequate to measure such a complex area. To better assess professionalism would require a combination of tools. But which combination would work best?

The collated definitions of professionalism were divided into five themes: adherence to ethical principles; effective interactions with patients and people important to patients; effective interactions with other people in health care; reliability; and commitment to autonomous maintenance and continuous improvement. The many assessment tools gleaned from a comb of the literature were organized according to their uses, which included evaluating observed clinical encounters, collating views of coworkers, recording unprofessional incidents and soliciting the opinions of patients.

In the end, after considering the components of professionalism and existing assessment techniques, Wilkinson and his colleagues came to the opinion that tools for observing behaviours of interest (via clinical and professionalism exercises) were crucial to the process. However, because people's behaviour will naturally be affected if they are aware of being observed, it is important to complement this information with the views of coworkers and patients.

Some areas of professionalism, such as moral reasoning, could be assessed through written exams or simulations. The assessment blueprint notes, though, that new tools must be developed to measure many aspects of professionalism, including reflectiveness, advocacy, dealing with uncertainty and life-long learning.
"The themes of professionalism that we have chosen are not the only way the cake could be cut, but we have attempted to synthesize the range of definitions and themes used by others into a unified whole," wrote Wilkinson and colleagues. "Over time, we anticipate that this classification could be challenged or refined. However, in the meantime, there is a pressing need to align these themes with assessment instruments."

The need for instruments to assess physician professionalism has also been recognized, to varying degrees, by medical licensing bodies in Canada. Though a formal assessment of professionalism doesn't appear to be a condition of licensing or licence renewal, some medical colleges mandate that doctors wishing to register with them demonstrate certain aspects of professionalism, such as continuing medical education. For example, the College of Physicians and Surgeons of British Columbia states in its bylaws that continuing professional development requirements must be met as a condition of revalidation of licensure (www.cpsbc.ca/files/u6/HPA-Bylaws.pdf \#nameddest=2-6).

Other colleges have developed systems that provide confidential feedback to physicians on their performance. The College of Physicians and Surgeons of Alberta, for instance, created the Physician Achievement Review program to solicit feedback on physician performance from patients and colleagues on various topics - some of which, such as communication skills, are related to professionalism (www.cpsa.ab.ca/Services /PARprogram/Overview.aspx). Similar programs have been adopted by the College of Physicians and Surgeons of Nova Scotia (www.nspar.ca) and the College of Physicians and Surgeons of Manitoba (http://mpar.ca/). The College of Physicians and Surgeons of Ontario is also exploring the value of such a program.

"With regard to the issue of multisource feedback, or 360 degree evaluation, it is generally a questionnairebased assessment method in which a person is evaluated on observable behaviours by the people who interact closely with him or her in the workplace," Kathryn Clarke, senior communications coordinator for the College of Physicians and Surgeons of Ontario, writes in an email, adding that, later this year or in early 2013, the college will launch a "pilot project with a group of 30-40 assessors to better understand the tools and use of multi-source feedback."

The colleges also have codes of conduct or guidelines regarding professional behaviour. For the most part, though, the behaviour of an individual physician is only scrutinized in detail when allegations of unprofessional conduct are made by patients. The discipline committee of the College of Physicians and Surgeons of Ontario, for one, addressed the following allegations in June 2012: sexual abuse of a patient (three cases), incompetence (two cases) and contravening a fee regulation (www.cpso.on.ca/whatsnew/committee schedule/default.aspx?id=1448).

In short, assessing professionalism in medicine is no easy task. Regardless of the complexities, however, there is one simple thing physicians can do to increase the odds that an assessment, however it is conducted, comes out in their favour - be nice. "Just plain, ordinary human courtesy and politeness goes a long way," says Wilkinson. "It's not really rocket science, is it?" Roger Collier, CMAJ

CMAJ 2012. DOI:10.1503/cmaj.109-4240

\section{More news online}

Educators propose "flipping" medical training: Medical educators are exploring a new model of instruction called the "flipped classroom" that reduces lectures and frees class time for discussion and interactive exercises in hopes of better preparing future physicians to be life-long learners (www.cmaj.ca /lookup/doi/10.1503/cmaj.109-4212). Lauren Vogel, CMAJ

Professionalism: Social media mishaps: It turns out that doctors who use Facebook, Twitter or other social media platforms are perfectly capable of embarrassing themselves and the medical profession as a whole - by posting unprofessional material (www.cmaj.ca/lookup/doi /10.1503/cmaj.109-4209). - Roger Collier, CMAJ 